Coherent structures and turbulence evolution in magnetized non-neutral plasmas

M. Romé, S. Chen, and G. Maero

Citation: AIP Conference Proceedings 1928, 020012 (2018);

View online: https://doi.org/10.1063/1.5021577

View Table of Contents: http://aip.scitation.org/toc/apc/1928/1

Published by the American Institute of Physics 


\title{
Coherent Structures and Turbulence Evolution in Magnetized Non-Neutral Plasmas
}

\author{
M. Romé ${ }^{1,2, a), b)}$, S. Chen ${ }^{3}$ and G. Maero ${ }^{1,2}$ \\ ${ }^{1}$ Dipartimento di Fisica, Università degli Studi di Milano, Via Celoria 16, 20133 Milano, Italy \\ ${ }^{2}$ INFN Sezione di Milano, Via Celoria 16, 20133 Milano, Italy \\ ${ }^{3}$ Institute of Fluid Physics, China Academy of Engineering Physics, Mianyang, Sichuan 621900, People's Republic \\ of China

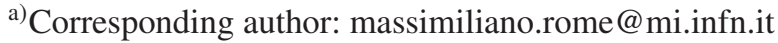 \\ b) URL: http://plasma.fisica.unimi.it
}

\begin{abstract}
The evolution of turbulence of a magnetized pure electron plasma confined in a Penning-Malmberg trap is investigated by means of a two-dimensional particle-in-cell numerical code. The transverse plasma dynamics is studied both in the case of free evolution and under the influence of non-axisymmetric, multipolar radio-frequency drives applied on the circular conducting boundary. In the latter case the radio-frequency fields are chosen in the frequency range of the low-order azimuthal (diocotron) modes of the plasma in order to investigate their effect on the insurgence of azimuthal instabilities and the formation and evolution of coherent structures, possibly preventing the relaxation to a fully-developed turbulent state.

Different initial density distributions (rings and spirals) are considered, so that evolutions characterized by different levels of turbulence and intermittency are obtained. The time evolution of integral and spectral quantities of interest are computed using a multiresolution analysis based on a wavelet decomposition of density maps. Qualitative features of turbulent relaxation are found to be similar in conditions of both free and forced evolution, but the analysis allows one to highlight fine details of the flow beyond the self-similarity turbulence properties, so that the influence of the initial conditions and the effect of the external forcing can be distinguished. In particular, the presence of small inhomogeneities in the initial density configuration turns out to lead to quite different final states, especially in the presence of competing unstable diocotron modes characterized by similar growth rates.
\end{abstract}

\section{INTRODUCTION}

Pure electron plasmas (or, more in general, plasmas with a single sign of charge) can be easily confined in PenningMalmberg traps [1], consisting of a set of coaxial, cylindrical conductors. Axial and radial confinement is provided by biasing two outer electrodes with respect to the innermost ones kept at ground, and by an (ideally uniform) axially directed magnetic field, respectively. The plasma confinement time may be limited by collisions of the electrons with the residual neutral gas at relatively high base pressures $\left(p>10^{-7} \mathrm{mbar}\right)$, or by asymmetries of the confining electric and magnetic fields under conditions of ultra-high vacuum $\left(p<10^{-8}\right.$ mbar) $[2,3]$. These asymmetries drag on the rotating plasma, decreasing its angular momentum and causing a radial expansion towards the trap walls. Nevertheless, very long confinement times can be achieved with the application of a rotating electric field on an azimuthally sectored electrode $[4,5]$.

Under typical experimental conditions, the time scales of the axial and the transverse motion of the electrons are well separated,

$$
\frac{2 \pi \epsilon_{0} B}{e n} \frac{(T / m)^{1 / 2}}{L} \gg 1,
$$

where $n, T$ and $L$ denote the plasma density, temperature and length, respectively, $B$ the magnetic field strength, $-e$ and $m$ the electron charge and mass, and $\epsilon_{0}$ the vacuum permittivity. In this case the bounce averaged non-collisional dynamics of the electron plasma can be accurately described by the drift-Poisson system (non-relativistic, cold fluid approximation) [6]

$$
\frac{\partial n}{\partial t}+\frac{1}{B} \mathbf{e}_{z} \times \nabla \phi \cdot \nabla n=0, \quad \nabla^{2} \phi=\frac{e n}{\epsilon_{0}},
$$


where $\phi$ is the electrostatic potential and $\mathbf{e}_{z}$ is the unit vector in the axial (magnetic field) direction. Eqs. (2) are isomorphic to the Euler equations for an inviscid, incompressible, uniform density, two-dimensional (2D) fluid [7], with vorticity $\zeta=e n / \epsilon_{0} B$ and stream function $\psi=\phi / B$.

Thanks to this analogy, strongly magnetized pure electron plasmas can therefore be used to investigate $2 \mathrm{D}$ fluid dynamics phenomena under accurately controlled and almost ideal conditions [8, 9, 10], possibly under the effect of external forcings (electric field drives) [11, 12, 13, 14], allowing for precise comparisons between experimental results and theoretical models. Experimental studies on (quasi-)2D fluid dynamics are typically performed using thin layers of conducting fluid [15], soap films [16, 17], or fluids in rapidly rotating tanks [18, 19], but in all these systems forcing and dissipation (due to friction of the fluid at the boundaries or to its internal viscosity) play a non-negligible role. Moreover, a precise measurement of vorticity, that provides a measure of the local angular velocity of the fluid, is in general a difficult task [20]. On the contrary, the electron plasma density (and therefore the corresponding fluid vorticity) can be easily measured by rapidly accelerating the electrons onto a phosphor screen held at a positive potential of a few $\mathrm{kV}$, and imaging their (axially-integrated) distribution with a charge coupled device camera.

Numerical simulations of the transverse dynamics of a pure electron plasma have been performed with different techniques, like, e.g., particle-in-cell (PIC) [21, 22, 23], fluid-Vlasov [24, 25], or contour dynamics [26] codes. Generally, these simulations reproduce with great accuracy the evolution of the electron density measured in the experiments, when condition (1) is satisfied and viscous/collisional effects may be neglected. Limitations to the 2D approximation have nonetheless been pointed out [27], and inherently three-dimensional cases have been also investigated [28, 29].

In an electron plasma the trigger for the subsequent non-linear evolution of the flow is the diocotron instability [7] (Kelvin-Helmholtz instability in the fluid dynamics context). The governing dynamical processes of the 2D inviscid flow evolution are the mutual advection of the vortices when they are well separated and their merger when they become sufficiently close to each other. It has to be noted that for an electron plasma the vorticity has a definite sign, so that classes of interactions in the evolution of the fluid system, like vortex scatterings, are excluded. On noncollisional time scales, the merger of vortices may lead to the formation of an intense central vortex superimposed on a weaker background vorticity resulting from filamentation and mixing, or to vortex crystals [30], i.e., long-lasting regular vortex patterns. To describe the formation of vortex crystals, the so-called "regional" maximum fluid entropy theory was proposed $[31,32]$. Here, intense vortices ergodically mix the background, which in turn cools their irregular motion, driving them into an equilibrium pattern. For the case of free relaxation, variational principles based on the minimization of enstrophy [33, 34] or the maximization of entropy [35, 36, 37, 25] have been also proposed. These statistical theories are based on the existence of global constraints (conserved integral quantities), and neglect the details of the early dynamical evolution of the flow.

On the other hand, a well estabilished (both numerically and experimentally) feature of 2D turbulent flows is the formation of large-scale, long-lived coherent structures or vortices arising from smaller-scale structures [38, 39]. In general, the vortex interactions depend on vortex size and circulation, distance between the vortex centers, background shear induced by the surrounding vortices [40, 41, 42, 22, 43], and all these features of the flow may strongly depend on the initial vorticity distribution $[44,39,45,46,47]$. The influence of the initial conditions in a pure electron plasma has been demonstrated computing the scaling properties of the freely relaxing 2D turbulence [48, 10, 49] from the analysis of experimental results obtained in different Penning-Malmberg traps [50, 25].

In order to identify the parameters playing a major role in the early dynamics of the system, the results of numerical simulations of the 2D electron plasma dynamics starting from particular classes of initial conditions (specifically, annular and spiral distributions) have been analyzed in Refs. [51, 52] in the case of free evolution and in Refs. [53, 54] in the case of forced turbulence, respectively. Both annular and spiral distributions are of experimental interest. Annular configurations are characterized in general by a low level of intermittency, as they typically lead to the formation of a small number of intense vortices in a tenuous turbulent background, whereas spiral distributions lead to a more complex dynamics and a higher level of intermittency due to the formation and subsequent merger of multiple vortices.

In particular, in the case of the free evolution of annular initial distributions a relation between the initial parameters of the ring and the time required to achieve a state of fully-developed turbulence has been evidenced [51]. These studies highlighted also the strong influence on the subsequent plasma evolution of density fluctuations introduced by the random number generator used to define the initial particle distribution. Even when using the same electron trapping time and the same injection parameters, uncontrolled inhomogeneities are present also in the experiments due to small variations of the conditions of the electron source or of the residual gas pressure, so that, for instance, the formation of vortex crystals and/or the relevant number of vortices turn out to strongly depend on the initial conditions $[45,10]$. The irreproducibility of the final state has been linked to the presence of different unstable diocotron modes, characterized by similar growth rates but slightly different energy contents determined by the fine details of 
the initial particle distribution [51].

In Ref. [53] PIC simulations of annular-shaped electron plasmas subjected to quadrupole and rotating-dipole excitations were presented. The analysis has been extended to higher multipoles and to spiral distributions in Ref. [54] with the aim to observe possible effects at the spatial scales of the involved unstable modes. These studies also present an experimental interest. Namely, radio-frequency (RF) drives are routinely used for the manipulation of non-neutral plasmas and the study of related particle transport phenomena $[4,55,56,57,58,59,60,61,62]$.

Here, the conclusions of Refs. [51, 52, 53, 54] are briefly reviewed, and results of new simulations and of the relevant statistical analysis are presented, that corroborate the previous findings. The attention is focused on the role on the late evolution of the system played by initial density fluctuations under the effect of a selected choice of multipolar RF drives, and by different initial conditions in the presence of a given external forcing. Perspectives of possible further investigations are also indicated.

\section{NUMERICAL SIMULATIONS}

The drift-Poisson equations (2) are integrated numerically using a 2D PIC code [63]. A Cartesian grid is adopted in the code and the electron charge density is computed on the grid nodes using a standard bilinear interpolation method. The boundary condition for the electrostatic potential is given by a function $\phi_{w}\left(r=R_{w}, \theta, t\right)$, where $(r, \theta)$ are polar coordinates defined in the circular domain, and $R_{w}$ is the radius of the conducting wall, suitably approximated by a piecewise linear function following the sides of the grid cells. The Poisson equation is solved by means of a 2D Fast Fourier Transform (FFT) combined with a capacity matrix.

Annular and Archimedean spiral density distributions are considered. Both kinds of density distribution are typically used in experiments on pure electron plasmas confined in Penning-Malmberg traps [64, 65, 25, 1, 66]. The density and the area covered by the initial electron distribution (and therefore the total number of particles) is the same in all simulations. The reference case is a flat annular density $n(r)=n_{0}\left[H\left(r-r_{-}\right)-H\left(r-r_{+}\right)\right]$, where $H$ denotes the Heaviside step function, and $r_{-}$and $r_{+}$are the inner and outer radius of the ring, respectively, with $r_{-} / R_{w}=0.3724$, $r_{+} / R_{w}=0.46$. The following parameters are fixed in all simulations: $n_{0}=10^{7} \mathrm{~cm}^{-3}, R_{w}=2 \mathrm{~cm}$, and $B=1$ $\mathrm{T}[67,68,51]$. In addition, the final time is $4 \mathrm{~ms}$, and the time step is $10^{-7} \mathrm{~s}$.

The simulations with external forcings reproduce experimentally relevant situations, where oscillating or rotating multipolar electric fields are applied on cylindrical electrodes splitted into several electrically insulated azimuthal sectors to control and manipulate the plasma dynamics $[4,5,69,70,56]$. More specifically, the conducting circular boundary is supposed to be split into four or eight azimuthal sectors (of angular aperture $\pi / 2$ and $\pi / 4$, respectively), where different potentials are applied. Rotating drives are simulated for a four-sectored electrode, considering $\pi / 2$ phase-shifted sinusoidally time varying potentials,

$$
\begin{gathered}
\phi_{w}^{\mathrm{co}, \mathrm{ctr}}(\theta, t)=V_{R F} \sum_{m=0}^{3} \cos \left(2 \pi v_{R F} t+\sigma m \pi / 2\right) \times[H(\theta-m \pi / 2)-H(\theta-(m+1) \pi / 2)] \\
=\frac{2 \sqrt{2}}{\pi} V_{R F} \sum_{n=1}^{\infty}\left\{\frac{1}{4 n-3} \cos \left[2 \pi v_{R F} t+\sigma(4 n-3) \theta-\sigma \frac{\pi}{4}\right]-\frac{1}{4 n-1} \cos \left[2 \pi v_{R F} t-\sigma(4 n-1) \theta-\sigma \frac{\pi}{4}\right]\right\},
\end{gathered}
$$

where $V_{R F}$ and $v_{R F}$ are the amplitude and frequency of the external RF drive, respectively, and $\sigma=-1$ and $\sigma=+1$ correspond to "co-" and "counter-"-rotating drives with respect to the electron plasma azimuthal motion, respectively. Non-rotating quadrupolar and octupolar excitations are also considered,

$$
\begin{aligned}
\phi_{w}^{\text {quad }}(\theta, t) & =V_{R F} \sin \left(2 \pi v_{R F} t\right) \sum_{m=0}^{3}(-1)^{m} H(\theta-m \pi / 2)=V_{R F} \sin \left(2 \pi v_{R F} t\right)\left\{\frac{1}{2}+\frac{2}{\pi} \sum_{n=1}^{\infty} \frac{1}{2 n-1} \sin [(4 n-2) \theta]\right\}, \\
\phi_{w}^{\text {octu }}(\theta, t) & =V_{R F} \sin \left(2 \pi v_{R F} t\right) \sum_{m=0}^{7}(-1)^{m} H(\theta-m \pi / 4)=V_{R F} \sin \left(2 \pi v_{R F} t\right)\left\{\frac{1}{2}+\frac{2}{\pi} \sum_{n=1}^{\infty} \frac{1}{2 n-1} \sin [(8 n-4) \theta]\right\}
\end{aligned}
$$

on four- and eight-sectored electrodes, respectively. The coupling of the RF drives with the plasma is expected to be effective mainly on the lowest order diocotron modes, i.e., density and potential perturbations with a spatial and temporal dependence of the form $\exp (i l \theta-i \omega t)$ with the integer $l$ denoting the azimuthal wavenumber, since the coefficients of the azimuthal Fourier expansion of the applied potential scale as $\left(r / R_{w}\right)^{l}$.

\section{EFFECT OF INITIAL DENSITY INHOMOGENEITIES}

As indicated above, in the PIC simulations the presence of unwanted fluctuations in the initial density distribution is practically unavoidable, because of the use of any type of random number generator. Such fluctuations reflect in 


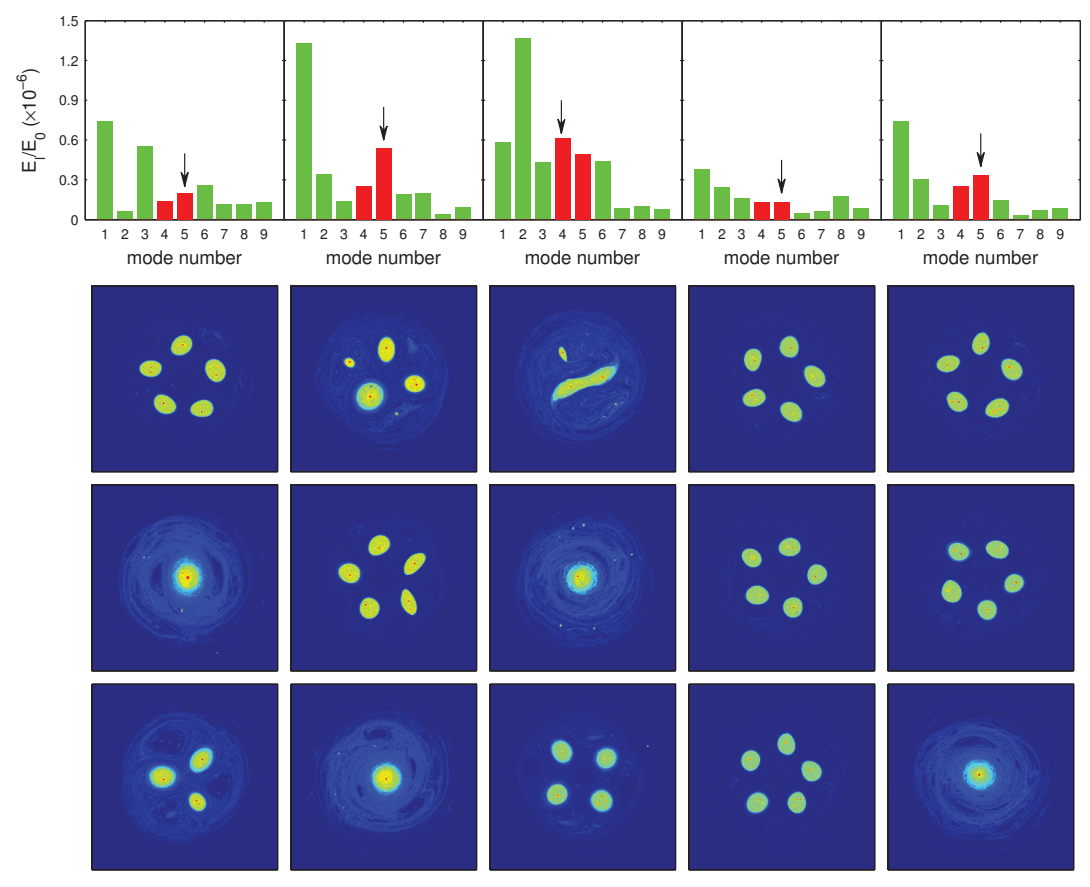

FIGURE 1. Results of five runs (along the columns) using different randomly generated initial distributions within a ring with $r_{-} / R_{w}=0.36$ and $r_{+} / R_{w}=0.45$. First row: Initial energies $E_{l}$ of the diocotron modes normalized to the initial energy $E_{0}$ of the $l=0$ mode; the dark/red bars correspond to the competing modes (the arrow indicates the mode with the largest initial energy) and the light/green ones to the other modes. Second, third and fourth row: Final states in the case of no external drive, a quadrupole drive with $V_{R F}=0.1 \mathrm{~V}$ and $v_{R F}=12.95 \mathrm{kHz}$ and a co-rotating drive with $V_{R F}=0.1 \mathrm{~V}$ and $v_{R F}=7.82 \mathrm{kHz}$, respectively.

an equivalent way the imperfections in real experiments, where particle distributions generated by any type of source have an intrinsically limited degree of uniformity and repeatability. Initial fluctuations may have a significant influence on the plasma evolution, even in the presence of an external forcing. In order to investigate this effect, all simulations have been repeated several times using different randomly generated initial electron positions. Due to the sensitive dependence of vortex merger events on the distribution of vorticity [53], the effects of initial fluctuations and other factors such as the growth of the diocotron modes are highly entangled, as it is shown in the following.

In the case of an annular initial distribution, an analysis of the early evolution of the flow allows one to estimate the growth rates of the diocotron modes [51]. At a given time $t$, the electron density and the electrostatic potential are calculated on a polar grid starting from the particle distribution, and Fourier analyzed as

$$
n(r, \theta, t)=\sum_{l=-\infty}^{+\infty} n_{l}(r) e^{i\left(l l \theta-\omega_{l} t\right)}, \quad \phi(r, \theta, t)=\sum_{l=-\infty}^{+\infty} \phi_{l}(r) e^{i\left(l \theta-\omega_{l} t\right)}
$$

where $\omega_{l}$ is in general a complex frequency, and $\gamma_{l}=\operatorname{Im}\left(\omega_{l}\right)>0$ defines the growth rate of the $l$-th mode, computed from an exponential fit of its (electrostatic) energy, computed as

$$
E_{l}(t)=-2 \pi \int_{0}^{1} \operatorname{Re}\left[n^{l}(r) \phi_{l}^{*}(r)\right] e^{2 \gamma_{l} t} r d r
$$

where the asterisk denotes the complex conjugate. In general, a fairly good agreement is obtained with the theoretical predictions of the growth rates based on a linear perturbation analysis [71].

The results of five runs relevant to an initial annulus with $r_{+} / R_{w}=0.45$ but using different randomly generated initial electron distributions are reported here in the case of free evolution and in the presence of two examples of (weak) external drives. The number of grid points used in the simulations is $N_{\text {grid }}$ in both Cartesian coordinates, with $N_{\text {grid }}=256$, and the number of macro-particles is $1.0 \cdot 10^{6}$. This choice of simulation parameters yields an initial distribution with more than 250 macro-particles/cell, thus satisfying the requirements of PIC codes and at the same 
time yielding an inhomogeneity of some percent with respect to the theoretical flat density. This is also the fluctuation level experimentally observed for some common types of electron sources (e.g., based on thermionic emission or RF excitation [58]). For the cases with a RF drive, $V_{R F}=0.10 \mathrm{~V}$, which corresponds to $\simeq 5 \%$ of (the absolute value of) the potential on the axis evaluated for the considered annular density profile in the case of grounded wall. This value of the perturbation amplitude is small enough for the system to be in a weak forcing regime, but sufficiently high to evidence the effect of the different drives. The frequencies of the RF drive are $v_{R F}=7.82 \mathrm{kHz}$ and $v_{R F}=12.95 \mathrm{kHz}$, which are close to the frequencies of the $l=3$ and $l=5$ diocotron modes, respectively, again for the considered annular density profile. The choice of drive frequencies close to low-order diocotron modes allows one to evidence whether a resonant interaction between the plasma and the applied perturbation is present and its influence on the evolution of turbulence.

The initial energies $E_{l}$ of the diocotron modes computed with Eq. (7) for $l=1-9$ (and normalized to the initial energy $E_{0}$ of the $l=0$ mode) are reported in the first row of Fig. 1 for the five randomly generated initial distributions. The competing modes (i.e., the unstable modes with similar growth rates) are indicated by darker (red) bars. The linear theory predicts that for the chosen configuration the diocotron modes with azimuthal number $l$ from 2 to 6 are unstable, with $l=5$ having the largest growth rate. In the other rows of Fig. 1 the final states of the runs are shown. In the case without RF drive (second row), three runs end up with five vortices, in agreement with their respective initial energy contents. However, two other runs end up with a smaller number of vortices, due to the occurrence of a greater number of vortex merger events during the flow evolution. In the presence of a quadrupole RF drive with $v_{R F}=12.95 \mathrm{kHz}$ (third row), runs No. 4 and 5 are almost unaffected by the weak external drive, and they still end up with five vortices. On the contrary, in run No. 1 the effect of the external RF drive is prevailing, and the initial annulus undergoes a full collapse. Such collapse turns out to be accelerated with respect to the previous case in run No. 3, while in run No. 2 the application of the drive helps to maintain the $l=5$ mode, and contrasting the effect of the competing $l=4$ mode that is evident in the previous case, resulting in a final state with five vortices. Similar features are also observed for the case of a co-rotating RF drive at $v_{R F}=7.82 \mathrm{kHz}$ (fourth row). In particular, in run No. 3 a final chain of four vortices is obtained instead of an almost fully collapsed state, indicating the survival of the initially dominant $l=4$ mode under the influence of the external drive. In other cases, like runs No. 1, 2 and 5, the drive seems able to excite an $l=3$ mode, and therefore the generation of a chain of three vortices, that is more prone to collapse to a single central patch.

\section{INFLUENCE OF INITIAL CONDITIONS AND DRIVES}

One of the characteristic features of 2D fluid turbulence is intermittency, i.e., the presence of field (here, vorticity) activity localized in both physical and spectral space. A suitable tool for its analysis is the wavelet decomposition [72, $73,65,74]$. Namely, in contrast to Fourier analysis wavelets have well localized basis functions both in physical and wavenumber space, allowing for the identification of local and non-stationary features of the flow. This is particularly relevant for the description of a fluid flow at high Reynolds numbers, which is characterized by the development of long-lived coherent structures with different spatial scales, that break the hypothesis of homogeneous turbulence.

In order to separate the coherent structures from the incoherent vorticity distribution and perform a distinct spectral analysis on the two components, a 2D orthogonal wavelet-based multiresolution analysis is adopted. Each density (vorticity) distribution saved during a numerical run is decomposed into coefficients that contain coarse and fine details at increasing resolution. These wavelet coefficients are then separated into coherent and incoherent parts using a suitable iterative method $[75,65]$. At each step of the iteration, the coefficients are divided into two classes, depending on whether their modulus is larger or smaller than a given threshold. The coherent vorticity field $\zeta_{>}$is reconstructed from the strongest wavelet coefficients by an inverse transform, while the incoherent vorticity field $\zeta_{<}$is obtained by subtracting the coherent from the total vorticity. The threshold is recomputed on the basis of the variance of the incoherent part and the procedure is repeated until the variation of the threshold becomes smaller than a given accuracy. The iteration method takes into account the inhomogeneity of the flow [51], i.e., the fact that the vorticity field is nonzero only in a fraction of the analysis domain (in the present case, of the cells of the grid used for the discretization of the system in the PIC code). In general, the coherent component of the flow can be reconstructed using only a small fraction (typically $<2 \%$ ) of the wavelet coefficients, but it contains the greatest part (typically $>95 \%)$ of the "energy of the signal", i.e., the enstrophy $Z \equiv(1 / 2) \int r d r d \theta \zeta^{2}$. The remaining small amplitude coefficients represent the incoherent component, and take into account the numerical noise and the presence of a low-level turbulent background. Although Eqs. (2) contain no viscous terms, the PIC method introduces an effective dissipation in the system due to coarse-graining, leading in general to a decay of all density moments (with the obvious 


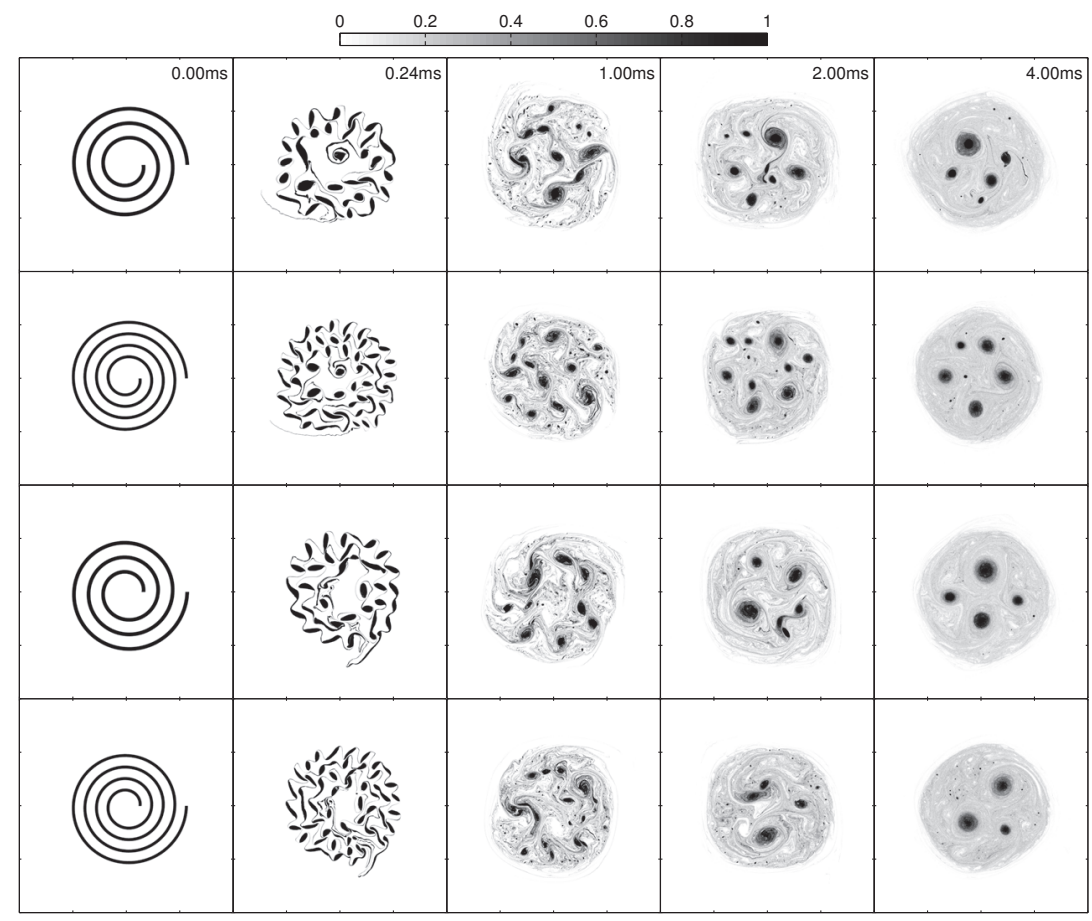

FIGURE 2. Electron density evolution for different initial configurations under the influence of an octupole drive with $V_{R F}=0.5$ $\mathrm{V}$ and $v_{R F}=5.145 \mathrm{kHz}$.

exception of the total number of particles), therefore including $Z$, as observed both in experiments and numerical simulations [21, 48, 52].

Depending on the initial density distribution, the evolution of the flow may be either dominated by the presence of persistent vortices (and possibly the formation of vortex crystal-like states), or by a higher turbulence level. The analysis is limited here to numerical simulations starting from different spiral configurations, but under the action of the same external forcing. In particular, an octupole drive with $V_{R F}=0.5 \mathrm{~V}$ is considered, with $v_{R F}=5.145 \mathrm{kHz}$, which is close to the frequency of the $l=2$ mode for the reference annular configuration.

Since a higher multipolar drive is used here, the spatial resolution of the PIC simulations is increased with respect to the cases analyzed above, in order to better detect possible effects at the spatial scales of the involved unstable modes. Consequently, in these simulations $N_{\text {grid }}=512$, and the number of macro-particles is $2.5 \cdot 10^{6}$. The evolution of the electron density is shown in Fig. 2. The initial configurations are a 3-turn clockwise (cw) spiral, a 4-turn cw spiral, a 3-turn counterclockwise (ccw) spiral and a 4-turn ccw spiral, respectively. The diocotron instability stage and the vortex merger events for these cases turn out to be quite different, but the effect of the drive is manifested through the generation of a square-shaped turbulent background in all cases, consistently with previous investigations [54]. The evolution of the total, coherent and incoherent enstrophy is shown in Fig. 3. Despite the differences in the initial configuration and in the details of the first stage of the evolution, the coherent parts show almost the same time variations for all cases, in accordance with previous observations [54]. On the other hand, the effect of the different initial configurations can still be identified from the incoherent parts. Namely, the cw-spirals lead to a slightly stronger turbulent background than ccw-spirals. A similar trend is also observed for other RF drive cases (not shown here).

The evolution of the coherent enstrophy spectra is shown in Fig. 4. The maximum wavenumber of the spectrum corresponds to the inverse of the minimum spatial scale of the wavelet functions. In turn, the latter is twice the length of a grid cell. In each successive decomposition level the spatial scale of the wavelet functions is increased by a factor of two and the wavenumber $k$ is accordingly reduced by the same factor. For the grid adopted in the present PIC simulations, the maximum level of wavelet decomposition is therefore $\left(\log _{2} N_{\text {grid }}-1\right)=8$. Analogously to the integral evolution, the enstrophy spectra show similar features for all cases. Moreover, due to the smallness of the vortex patches formed at the end of diocotron instability stage, the difference in spatial scale between the coherent and incoherent parts is less distinguishable, resulting simply in a broadening of the contour ridges instead of a bifurcation 

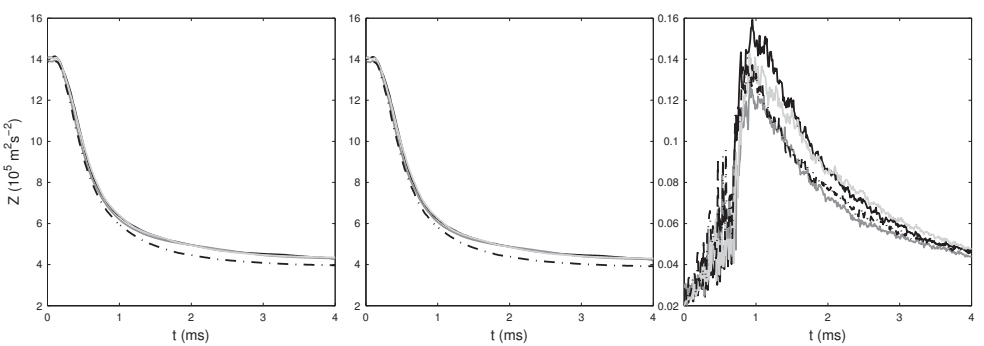

FIGURE 3. Total (left), coherent (center) and incoherent (right) enstrophy evolution for the same initial configurations and the same external drive as in Fig. 2. Black solid, dark gray solid, light gray solid and black dash-dotted lines correspond to a 3-turn cw-, 4-turn cw-, 3-turn ccw- and 4-turn ccw-spiral initial distribution, respectively.

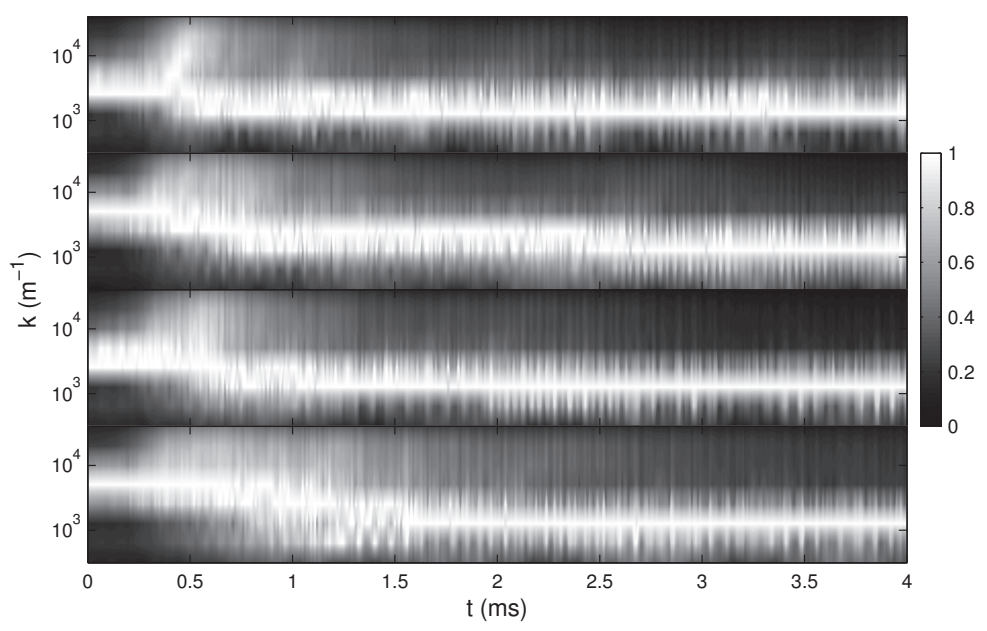

FIGURE 4. Evolution of the coherent enstrophy spectrum (normalized by its maximum value at each time) for the same initial configurations and the same external drive as in Fig. 2. The rows correspond to a 3-turn cw-, 4-turn cw-, 3-turn ccw- and 4-turn ccw-spiral initial distribution, respectively.

structure as more clearly evidenced for the case of free evolution both for annular and spiral configurations [51, 52]. In order to investigate complementary or additional information, the wavelet decomposition technique is applied also to the energy distribution (calculated from the density and the electrostatic potential maps). Within the frame of the drift-Poisson equations, the energy of the system is purely electrostatic,

$$
E=-e \int n\left(\frac{1}{2} \phi_{p}+\phi_{v}\right) r d r d \theta
$$

where $\phi_{p}(r, \theta, t)$ is the plasma potential, satisfying the Poisson equation with the boundary condition $\phi_{p}\left(R_{w}, \theta, t\right)=0$, and $\phi_{v}(r, \theta, t)$ denotes the vacuum potential satisfying the Laplace equation with $\phi_{v}\left(R_{w}, \theta, t\right)=\phi_{w}(\theta, t)$.

The evolution of the electrostatic energy spectra for the considered spiral initial configurations is shown in Fig. 5. Since the charge center of the initial spiral distributions does not coincide with the trap axis, an inherent $l=1$ mode is present in all cases. The motion of the charge center causes regular variations observed in the energy spectra. The time interval between the maximum values at both large or small scales is approximately $1 \mathrm{~ms}$, in agreement with the plasma bulk rotation period. Furthermore, there is a regular phase shift between the ccw- and cw-spiral distributions, indicated by the different time of occurrence of the maximum values at small scales. This observation points to a different energy coupling between the electron plasma and the external RF drive due to the different angular frequency profile, and/or to its shear profile, in the initial plasma configuration. 


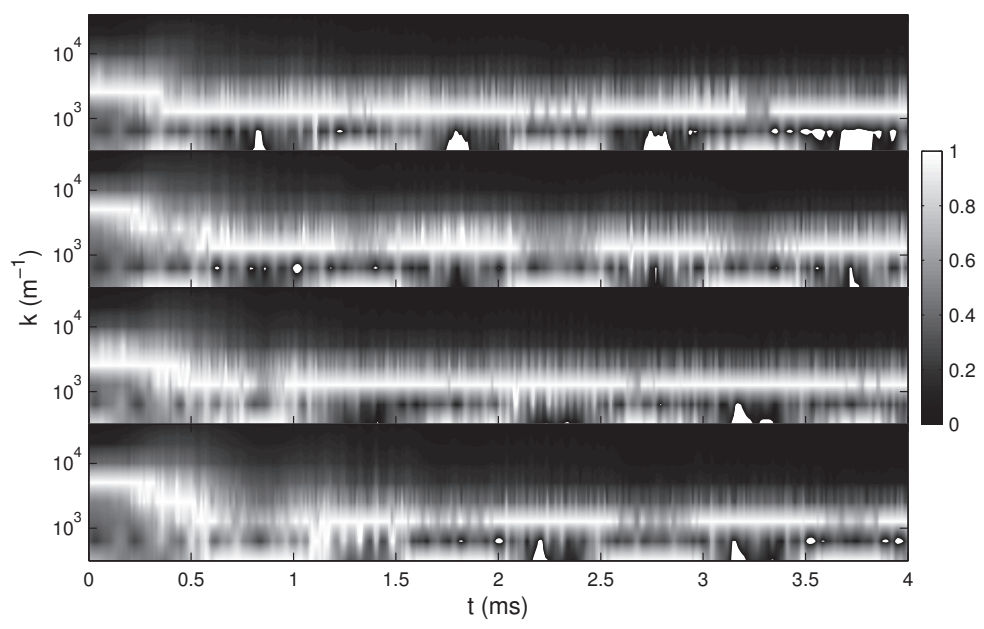

FIGURE 5. Evolution of the electrostatic energy spectrum (normalized by its maximum value at each time) for the same initial configurations and the same external drive as in Fig. 2. The rows correspond to a 3-turn cw-, 4-turn cw-, 3-turn ccw- and 4-turn ccw-spiral initial distribution, respectively.

\section{CONCLUSIONS}

The formation of coherent structures and the evolution of 2D turbulence have been subject of investigation for decades in the fluid dynamics community. In particular, the 2D turbulence in pure electron plasmas has been studied by variational principles [34, 31, 25], time scaling of the vortices present in the flow [32], statistical techniques [10, 49, 52]. The statistical theories rely on the presence of global constraints, and usually neglect the details of the early dynamical evolution of the flow. On the other hand, the initial vorticity distribution may affect the subsequent vortex interactions by determining vortex size, vorticity amplitude and separation of the vortex centers in the early stage of the flow. The aim of the present work and of the previous investigations reported in the literature $[51,52,53,54]$ is to characterize, by means a systematic variation of the initial conditions and of the characteristics of forcing, the parameters playing a major role in the early dynamics of the flow leading to an evolution dominated by coherent vortices (leading eventually to the formation of vortex crystal-like states), or by a higher turbulence level, and to determine quantitative indices that may help to characterize the features of the turbulence.

The frequencies and the spatial structure of the applied electric drives, and the initial density configurations (rings and spirals) considered in the simulations are relevant to typical experiments performed on electron plasmas confined in Penning-Malmberg traps. The investigation is performed by means of a wavelet-based multiresolution analysis of the vorticity and by the study of the time evolution of a few relevant integral quantities (specifically, enstrophy and electrostatic energy) and their spectra. In particular, thanks to their localization both in physical and wavenumber space, wavelets allow for better discrimination with respect to the Fourier analysis of evolving coherent structures with different spatial scales in the flow, and therefore for a more accurate and detailed description of intermittency phenomena. The wavelet analysis allows one to clearly relate the presence of time periods characterized by a decreasing coherent enstrophy and the existence of bifurcation structures in the relevant spectra (with the transfer of enstrophy to both large and small spatial scales) to the occurrence of (possibly repeated) stages of vortex merger processes. A relation between the initial conditions and the time required to achieve a state of fully developed turbulence has been shown in the free evolution of annular [51] and spiral [52] distributions.

The early dynamics of the system is obviously determined by the evolution of the diocotron instability. In the case of annular initial distributions, vortex merging processes dominate the plasma evolution up to the formation of a single central vortex when low-order diocotron modes are dominant, while in the opposite case vortex merging processes are largely suppressed, and the initially dominant mode typically leads to the formation of a chain of vortices (vortex crystal). These features are manifested in the evolution of the enstrophy spectra by abrupt changes or bifurcations in the spatial scales corresponding to maximum enstrophy content. The azimuthal symmetry of an applied weak external perturbation influences the shape of the turbulent backgrounds (e.g., square shapes are found in the case of octupole $\mathrm{RF}$ drives, as reported also here). 
Inhomogeneities in the initial density profile are introduced by the random number generator used to define the initial particle distribution in the numerical simulations, or by small variations of the electron source conditions in the experiments, and play a dramatic role in determining whether a single patch equilibrium state can be reached or the relaxation is temporarily or indefinitely halted by the occurrence of intermittent phenomena such as localized vortices. The irreproducibility of the final state has been related to the presence of multiple unstable modes with similar growth rates but slightly different energy contents [51], both in the free and in the forced evolution, as confirmed also by the results of the simulations reported here.

In the case of spiral-like initial distributions the dynamics is inherently more complicated and characterized by a stronger intermittency, with multiple vortices and frequent merger events, both in the case of free [53] and forced evolution [54] as confirmed also in the present work. In general, the time frequency and spatial scales associated to the peak of the energy spectrum are related to the $l=1$ diocotron mode that is present already in the initial condition due to the asymmetry of the spiral configuration. This mode gives therefore the dominant contribution to the energy present and injected into the system. Only small differences in the time evolution are detected due to the particular drive applied to the system, but the intermittency levels for the considered amplitude and frequencies of forcings (the latter corresponding to the low-order diocotron modes) are very similar.

In perspective, more insight on the turbulent evolution of an electron plasma may come from the study of the role of the turbulent background, the influence of the distribution and concentration of a second population of charged particles, the effect of higher-frequency and/or higher-amplitude drives, the effect of dissipation (e.g., resistive loads on the trap wall).

\section{ACKNOWLEDGMENTS}

This work is partially supported by NSFC (No. 11505172) and INFN Group V ("PLASMA4BEAM4" project).

\section{REFERENCES}

[1] J. H. Malmberg and J. S. deGrassie, Phys. Rev. Lett. 35, p. 577 (1975).

[2] D. H. E. Dubin, A. A. Kabantsev, and C. F. Driscoll, Phys. Plasmas 19, p. 056102 (2012).

[3] D. L. Eggleston, Phys. Plasmas 19, p. 042307 (2012).

[4] X.-P. Huang, F. Anderegg, E. M. Hollmann, C. F. Driscoll, and T. M. O’Neil, Phys. Rev. Lett. 78, p. 875 (1997).

[5] E. M. Hollmann, F. Anderegg, and C. F. Driscoll, Phys. Plasmas 7, p. 2776 (2000).

[6] C. F. Driscoll and K. S. Fine, Phys. Fluids B 2, p. 1359 (1990).

[7] R. H. Levy, Phys. Fluids 8, p. 1288 (1965).

[8] C. F. Driscoll, D. A. Schecter, D. Z. Jin, D. H. E. Dubin, K. S. Fine, and A. C. Cass, Phys. A 263, p. 284 (1999).

[9] D. Durkin and J. Fajans, Phys. Fluids 12, p. 289 (2000).

[10] M. Romé and F. Lepreti, Eur. Phys. J. Plus 126, p. 38 (2011).

[11] J. Fajans, E. Y. Backhaus, and J. E. McCarthy, Phys. Plasmas 6, p. 12 (1999).

[12] J. Fajans, E. Gilson, and L. Friedland, Phys. Rev. Lett. 82, p. 4444 (1999).

[13] K. Gomberoff, H. Higaki, K. Ito, and H. Okamoto, Phys. Rev. E 94, p. 043204 (2016).

[14] N. C. Hurst, J. R. Danielson, D. H. E. Dubin, and C. M. Surko, Phys. Rev. Lett. 117, p. 235001 (2016).

[15] J. Sommeria, J. Fluid Mech. 170, p. 139 (1986).

[16] M. Rivera, P. Vorobieff, and R. E. Ecke, Phys. Rev. Lett. 81, p. 1417 (1998).

[17] M. A. Rutgers, Phys. Rev. Lett. 81, p. 2244 (1998).

[18] J. Sommeria, S. D. Meyers, and H. L. Swinney, Nature 331, p. 689 (1988).

[19] C. N. Baroud, B. B. Plapp, Z. S. She, and H. L. Swinney, Phys. Rev. Lett. 88, p. 114501 (2002).

[20] J. M. Wallace and J. F. Foss, Ann. Rev. Fluid Mech. 27, p. 469 (1995).

[21] D. A. Schecter, D. H. E. Dubin, K. S. Fine, and C. F. Driscoll, Phys. Fluids 11, p. 4162 (1999).

[22] M. Amoretti, D. Durkin, J. Fajans, R. Pozzoli, and M. Romé, Phys. Plasmas 8, p. 3865 (2001).

[23] G. L. Delzanno, G. Lapenta, and J. M. Finn, IEEE Trans. Plasma Sci. 30, p. 34 (2002).

[24] M. Romé, M. Brunetti, F. Califano, F. Pegoraro, and R. Pozzoli, Phys. Plasmas 7, p. 2856 (2000).

[25] D. J. Rodgers, S. Servidio, W. H. Matthaeus, D. C. Montgomery, T. B. Mitchell, and T. Aziz, Phys. Rev. Lett. 102, p. 244501 (2009). 
[26] G. G. M. Coppa, F. Peano, and F. Peinetti, J. Comp. Phys. 182, p. 392 (2002).

[27] A. J. Peurrung and J. Fajans, Phys. Fluids B 5, p. 4295 (1993).

[28] Yu. Tsidulko, R. Pozzoli, and M. Romé, J. Comp. Phys. 209, p. 406 (2005).

[29] K. Gomberoff, J. Wurtele, A. Friedman, D. P. Grote, and J. Vay, J. Comp. Phys. 225, p. 1736 (2007).

[30] K. S. Fine, A. C. Cass, W. G. Flynn, and C. F. Driscoll, Phys. Rev. Lett. 75, p. 3277 (1995).

[31] D. Z. Jin and D. H. E. Dubin, Phys. Rev. Lett. 80, p. 4434 (1998).

[32] D. Z. Jin and D. H. E. Dubin, Phys. Rev. Lett. 84, p. 1443 (2000).

[33] C. E. Leith, Phys. Fluids 27, p. 1388 (1984).

[34] X.-P. Huang and C. F. Driscoll, Phys. Rev. Lett. 72, p. 2187 (1994).

[35] J. Miller, Phys. Rev. Lett. 65, p. 2137 (1990).

[36] R. Robert and J. Sommeria, J. Fluid Mech. 229, p. 291 (1991).

[37] J. Miller, P. B. Weichman, and M. C. Cross, Phys. Rev. A 45, p. 2328 (1992).

[38] J. C. McWilliams, J. Fluid Mech. 146, p. 21 (1984).

[39] P. Santangelo, R. Benzi, and B. Legras, Phys. Fluids A 1, p. 1027 (1989).

[40] M. V. Melander, N. J. Zabusky, and J. C. McWilliams, J. Fluid Mech. 195, p. 303 (1988).

[41] D. G. Dritschel and D. W. Waugh, Phys. Fluids A 4, p. 1737 (1992).

[42] D. W. Waugh, Phys. Fluids A 4, p. 1745 (1992).

[43] R. R. Trieling, O. U. Velasco Fuentes, and G. J. F. van Heijst, Phys. Fluids 17, p. 087103 (2005).

[44] R. Benzi, S. Patarnello, and P. Santangelo, J. Phys. A 21, p. 1221 (1988).

[45] C. F. Driscoll, D. Z. Jin, D. A. Schecter, E. J. Moreau, and D. H. E. Dubin, Phys. Scripta T84, p. 76 (2000).

[46] C. Kalelkar, Phys. Rev. E 72, p. 056307 (2005).

[47] L. J. A. van Bokhoven, R. R. Trieling, H. J. H. Clerckx, and G. J. F. van Heijst, Phys. Fluids 19, p. 046601 (2007).

[48] D. J. Rodgers, W. H. Matthaeus, T. B. Mitchell, and D. C. Montgomery, Phys. Rev. Lett. 105, p. 234501 (2010).

[49] F. Lepreti, M. Romé, G. Maero, B. Paroli, R. Pozzoli, and V. Carbone, Phys. Rev. E 87, p. 063110 (2013).

[50] M. Amoretti, G. Bettega, F. Cavaliere, M. Cavenago, F. De Luca, R. Pozzoli, and M. Romé, Rev. Sci. Instrum. 74, p. 3991 (2003).

[51] S. Chen, G. Maero, and M. Romé, J. Plasma Phys. 81, p. 495810511 (2015).

[52] M. Romé, S. Chen, and G. Maero, Plasma Sources Sci. Technol. 25, p. 035016 (2016).

[53] M. Romé, S. Chen, and G. Maero, Plasma Phys. Control. Fusion 59, p. 014036 (2017).

[54] S. Chen, G. Maero, and M. Romé, J. Plasma Phys. 83, p. 705830303 (2017).

[55] J. R. Danielson, T. R. Weber, and C. M. Surko, Phys. Plasmas 13, p. 123502 (2006).

[56] G. Bettega, B. Paroli, R. Pozzoli, and M. Romé, J. Appl. Phys. 105, p. 053303 (2009).

[57] B. Paroli, F. De Luca, G. Maero, R. Pozzoli, and M. Romé, Plasma Sources Sci. Technol. 19, p. 045013 (2010).

[58] G. Maero, B. Paroli, R. Pozzoli, and M. Romé, Phys. Plasmas 18, p. 032101 (2011).

[59] A. A. Kabantsev and C. F. Driscoll, AIP Conf. Proc. 1668, p. 020002 (2015).

[60] B. Paroli, G. Maero, R. Pozzoli, and M. Romé, Phys. Plasmas 21, p. 122102 (2014).

[61] G. Maero, S. Chen, R. Pozzoli, and M. Romé, J. Plasma Phys. 81, p. 495810503 (2015).

[62] G. Maero, B. Paroli, R. Pozzoli, and M. Romé, J. Instrum. 11, p. C09007 (2016).

[63] G. Maero, M. Romé, F. Lepreti, and M. Cavenago, Eur. Phys. J. D 68, p. 277 (2014).

[64] A. J. Peurrung and J. Fajans, Phys. Fluids A 5, p. 493 (1993).

[65] G. Bettega, R. Pozzoli, and M. Romé, New J. Phys. 11, p. 053006 (2009).

[66] J. M. Kriesel and C. F. Driscoll, Phys. Plasmas 5, p. 1265 (1998).

[67] M. Maggiore, M. Cavenago, M. Comunian, F. Chirulotto, A. Galatà, M. De Lazzari, A. M. Porcellato, C. Roncolato, S. Stark, A. Caruso, A. Longhitano, F. Cavaliere, G. Maero, B. Paroli, R. Pozzoli, and M. Romé, Rev. Sci. Instrum. 85, p. $02 B 909$ (2014).

[68] M. Romé, F. Cavaliere, M. Cavenago, S. Chen, and G. Maero, AIP Conf. Proc. 1668, p. 030001 (2015).

[69] G. Bettega, F. Cavaliere, B. Paroli, M. Cavenago, R. Pozzoli, and M. Romé, Phys. Plasmas 14, p. 102103 (2007).

[70] G. Bettega, F. Cavaliere, B. Paroli, R. Pozzoli, M. Romé, and M. Cavenago, Phys. Plasmas 15, p. 032102 (2008).

[71] R. C. Davidson, An Introduction to the Physics of Nonneutral Plasmas (Addison-Wesley, Redwood City, 1990).

[72] M. Farge, Ann. Rev. Fluid Mech. 24, p. 395 (1992).

[73] S. G. Mallat, A wavelet tour of signal processing (Academic Press, San Diego, 1999).

[74] B. Paroli, G. Bettega, G. Maero, M. Romé, M. Norgia, A. Pesatori, , and C. Svelto, Rev. Sci. Instrum. 81, p. 063503 (2010).

[75] M. Farge, K. Schneider, and N. Kevlahan, Phys. Fluids 11, p. 2187 (1999). 